\title{
Co-Incidence of Epstein-Barr Virus and High-Risk Human Papillomaviruses in Cervical Cancer of Syrian Women
}

Hamda Al-Thawadi*1, Lina Ghabreau*2,3, Tahar Aboulkassim ${ }^{4}$, Amber Yasmeen $^{4}$, Semir Vranic $^{1}$, Gerald Batist ${ }^{4,5}$ \& Ala-Eddin Al Moustafa ${ }^{1,3,5,6}$

${ }^{1}$ College of Medicine, Qatar University, Doha, Qatar; ${ }^{2}$ Faculty of Medicine, Pathology Department, University of Aleppo \& ${ }^{3}$ Syrian Research Cancer Centre of the Syrian Society against Cancer, Aleppo, Syria; ${ }^{4}$ Segal Cancer Centre, Lady Davis Institute for Medical Research of the Sir Mortimer B. Davis-Jewish General Hospital \& ${ }^{5}$ Oncology Department, McGill University, Montreal, Quebec, Canada; ${ }^{6}$ Biomedical Research Centre, Qatar University, Doha, Qatar.

Key words: EBV, High-risk HPV, Cervical cancer, Syrian women, Cancer phenotype

Correspondence should be addressed to Ala-Eddin Al Moustafa, College of Medicine

Qatar University

Doha, Qatar

Tel: 97444037817

E-mail: aalmoustafa@qu.edu.qa; ala-eddin.almoustafa@mcgill.ca

*These authors contributed equally to this work. 


\begin{abstract}
Epstein-Barr virus (EBV) has been recently shown to be co-present with high-risk human papillomaviruses (HPVs) in human cervical cancer; thus, these oncoviruses play an important role in the initiation and/or progression of this cancer. Accordingly, our group has recently viewed the presence and genotyping distribution of high-risk HPVs in cervical cancer in Syrian women; our data pointed out that HPVs are present in $95.45 \%$ of our samples. Herein, we aim to explore the co-prevalence of EBV and high-risk HPVs in 44 cervical cancer tissues from Syrian women using polymerase chain reaction (PCR), immunohistochemistry (IHC) and tissue microarray (TMA) analyses. We found that EBV and high-risk HPVs are co-present in 15/44 (34\%) of the samples. Additionally, we report that the co-expression of LMP1 and E6 genes of EBV and high-risk HPVs, respectively, is associated with poorly differentiated squamous cell carcinomas phenotype; this is accompanied by a strong and diffused ld-1 overexpression, which is an important regulator of cell invasion and metastasis. These data imply that EBV and HPVs are copresent in cervical cancer in the Middle East area including Syria and their co-presence is associated with a more aggressive cancer phenotype. Future investigations are needed to elucidate the exact role of EBV and HPVs cooperation in cervical carcinogenesis.
\end{abstract}

Key words: Cervical cancer - viruses - Human Papillomaviruses - Epstein-Barr virus Id-1 


\section{Introduction}

Cervical cancer is the fourth most common malignancy amongst women worldwide with approximately 528,000 new cases and 266,000 deaths each year estimated by the World Health Organization. Notably, most cervical cancer deaths (87\%) occur in the developing countries. Currently, it is well known that the majority of cancer deaths are the result of metastasis, either directly due to tumor involvement of critical organs or indirectly due to therapeutic resistance and the inability of available therapy to control tumor progression 1. On the other hand, it is estimated that approximately $20 \%$ of human cancers could be linked to oncoviruses infection including Epstein-Barr virus (EBV) and high-risk human papillomaviruses (HPV) especially types 16, 18 and 33 2-4. EBV is a human gammaherpesvirus that infects more than $90 \%$ of the human adult population. Acute infection with EBV can cause infectious mononucleosis, and its latent state can lead to several types of human B-cell lymphomas and carcinomas, especially nasopharyngeal ${ }^{5-}$ 6 .

Today, it is well established that high-risk of HPVs infections are important etiological factors in the development of human cervical cancer; as more than $96 \%$ of cervical cancers are positive for high-risk HPVs especially types 16, 18, 31, 33 and 35 worldwide including the Middle East region ${ }^{3,7}$. Moreover, accumulating evidence suggests that persistent infection with these viruses is necessary for cervical precursors to evolve into invasive carcinomas ${ }^{8}$. Accordingly, we have explored the presence of high-risk HPVs in cervical cancer in Syrian women; our study revealed that $95 \%$ of our samples are positive for HPVs; more significantly, we noted that the most frequent high-risk HPV types in Syrian women are 33, 16, 18, 45, 52, 58 and 35, in descending order. Furthermore, the expression of E6 onco-protein of high-risk HPVs was found to be correlated with the overexpression of Id-1, which is a member of the Inhibitor of DNA-binding (ID) proteins 9 .

ID proteins constitute a family of highly preserved transcriptional controllers that play critical roles during normal development and in the maintenance of homeostasis in human tissue ${ }^{10}$. The main biological properties of ID proteins are inhibition of differentiation and 
conservation of the self-renewal capability and multipotency of stem cells ${ }^{11}$. ID proteins are overexpressed in several human carcinomas ${ }^{11-12}$. More specifically, Id-1 protein expression is directly involved in cancer initiation and/or progression in different types of human malignancies including cervical ${ }^{9,13-15}$. On the other hand, it has been pointed out that LMP1 onco-protein of EBV up-regulates Id-1 expression in nasopharyngeal immortalized and cancer cells ${ }^{16-17}$; however, the association between EBV onco-proteins and Id-1 in human carcinomas, including cervical is not clear.

Earlier studies have indicated that EBV is frequently present in human cervical cancer tissues, suggesting EBV is associated with the development of cervical cancer ${ }^{18}$. Moreover, it has been shown that the co-occurrence of EBV and high-risk HPVs in cervical tissues is more frequent in patients with high-grade squamous intraepithelial lesions in comparison with low-grade lesions ${ }^{19}$. Thus, the presence of EBV in high-grade cervical lesions and cancer could suggest a possible cooperation between EBV and HPV in human cervical carcinogenesis; however, there are no studies regarding the copresence of EBV and HPVs in the Middle East region.

Therefore, in this study, we evaluated the co-presence of these viruses and their association with Id-1 expression in cervical cancers in Syrian women. Our study pointed out that EBV and high-risk HPVs are co-present in $34 \%$ of our samples; more significantly, we noted that the co-incidence of these viruses is associated with poorly differentiated squamous cell carcinomas, which is accompanied with ld-1 overexpression. 


\section{Results}

We have recently explored the presence of high-risk HPVs in a cohort of 44 cervical cancer samples from Syrian women. Our study revealed that $42(95.45 \%)$ of the 44 samples are high-risk HPVs positive. Moreover, these data revealed that the most prevalent high-risk HPV types are 33, 16, 18, 45, 52, 58, 35, 51 and $31^{9}$ in descending order. Herein, we investigated the co-presence of EBV and high-risk HPVs in our 44 samples by polymerase chain reaction (PCR) and immunohistochemistry (IHC) analysis using specific primers for LMP1 and EBNA1 as well as E6/E7 genes of EBV and HPVs, respectively, (Table 1) and monoclonal antibodies (mAbs) for LMP1 and E6, as described in the Materials and Methods section. We found that $15(34 \%)$ of the 44 samples are positive ( $\geq 1 \%$ positive cancer cells) for both EBV and high-risk HPVs (Table 2 and Figure 1A-D).

Next, we assessed the association between the co-presence of these viruses and tumor phenotype in our samples using tissue microarray (TMA) methodology. Our data indicate that the co-expression of the LMP1 and E6 onco-proteins of EBV and high-risk HPVs, respectively, is associated with poorly differentiated squamous cell carcinoma form (Figure 2) in comparison with positive cases of EBV alone or HPVs alone as well as negatives cases for both, EBV and HPVs. On the other hand, we noted that the expression of LMP1 is located in cervical squamous cell carcinomas and frequently in stromal cells in addition to tumor infiltrating lymphocytes (Figure 1D); however, E6 of HPV, in general, is detected in cancer cells while the stromal and inflammatory cells (lymphocytes) are negative (Figures 1D and 2).

Finally, we explored the association between the presence of EBV and HPVs with Id-1 overexpression in our Syrian samples using IHC. We found that the co-expression of LPM1 and E6 (of EBV and HPV, respectively) is associated with diffused and strong ld-1 over-expression in all invasive squamous cell carcinomas including high-grade carcinomas (Figure 2). 


\section{Discussion}

In this investigation, we explored, for the first time, the co-presence of EBV and high-risk HPVs in human cervical cancer and the role of this co-incidence with cancer phenotype in the conventional Middle East region. While, one study from North Africa pointed out that EBV and high-risk HPVs are co-present in $67.2 \%$ of cervical cancer cases in Algerian women ${ }^{20}$. Herein, it is important to highlight that infection with, at least one high-risk HPV alone, is necessary but not sufficient to provoke cervical cancer initiation, additional oncovirus infection and/or host genetic changes are required to drive neoplastic transformation and consequently lead to tumor formation ${ }^{21-22}$. In our investigation, we demonstrated that EBV is co-present with high-risk HPVs in $34 \%$ of cervical cancer cases in the Syrian population. Accordingly, a recent meta-analysis study of 25 investigations regarding the presence of EBV in human cervical cancer revealed that EBV is present in $43.63 \%$ of samples from cancer patients in comparison with $19 \%$ of samples from heathy people or patients with cervical intraepithelial neoplasia grade 1 (CIN) (27.34\%) or CIN grade $2 / 3(34.67 \%){ }^{19}$. More significantly, co-infection with EBV and HPV is present in most of the cases, which display a similar phenotype of EBV infection ${ }^{19}$; moreover, EBV infection is associated with differentiation (grade) of cervical epithelial cells ${ }^{18}$. On the other hand, it has been pointed out that cervical carcinomas are four times more likely to occur among EBV positive patients as compared with patients without EBV infection ${ }^{19}$, which suggests a strong cooperation between EBV and HPVs in cervical carcinogenesis and possibly other human carcinomas ${ }^{5}$. This concurs with our findings regarding the copresence of EBV and high-risk HPVs and their association with cervical carcinomas in all positive cases, all of which are high grade invasive cancers. Likewise, we have recently reported that EBV and high-risk HPVs are co-present in 32\% of human breast cancer samples and their co-presence is associated with high-grade breast carcinomas and positive axillary lymph nodes ${ }^{23}$.

On the other hand, it is important to highlight that EBV onco-proteins' expression in cervical tissues is still controversial. Using in situ techniques for the detection of viral genomes or gene expressions, few investigations showed that EBV is present in cervical 
carcinoma cells 20, 24-26. However, others studies reported EBV localization in infiltrating lymphoid cells next to cervical carcinomas and concluded that EBV infection could not play a specific role in cervical carcinogenesis ${ }^{27-28}$. Interestingly, our study revealed that the expression of LMP1 protein is present in cervical squamous cell carcinomas and occasionally in the stroma as well as in tumor infiltrating lymphocytes; LMP1 is co-present with E6 onco-protein of high-risk HPVs in cervical carcinoma cells in most cases.

Concerning the association between the two oncoviruses (EBV and HPV) and Id-1 gene, which is overexpressed in several human carcinomas, it has been reported that LMP1 onco-protein of EBV up-regulates the expression of Id-1 but not FoxO3a in human Hodgkin's lymphoma cells ${ }^{29}$. Likewise, in nasopharyngeal carcinoma, LMP1 induces an upregulation of Id-1 via $\mathrm{FoxO} 3 \mathrm{a}$ inactivation ${ }^{30}$. However, there is no studies regarding the EBV onco-proteins and Id-1 in human cervical cancer. In our present report, we demonstrate for the first time, the co-expression of LMP1 and E6 of EBV and high-risk HPVs, respectively, which is associated with Id-1 overexpression in human cervical cancer samples. However, herein, it is important to highlight that few investigations, including one from our lab, have pointed out that the presence of E6/E7 of high-risk HPVs is linked with Id-1 overexpression in human cervical cancer cells ${ }^{9,15,31}$. More significantly, we have demonstrated that E6/E7 onco-proteins of HPV type 16 bind and active Id-1 promotor in human breast cancer cells; in parallel, we reported that Id-1 is the main regulator of cell invasion and metastasis induced by E6/E7 onco-proteins in these cancer cells ${ }^{32}$. Accordingly, it is possible that EBV and high-risk HPV cooperate to up-regulate the expression of Id-1 in human cervical cancer, which could enhance rapidly the progression of this cancer into invasive and metastatic form.

Nevertheless, further studies are necessary to clarify the role and pathogenesis of the copresence of EBV and HPVs in human cervical carcinomas; especially since EBV and HPVs vaccines are presently under clinical trial and available, respectively ${ }^{33-35}$. This is an important step, which could possibly limit cervical cancer initiation as well as its progression to a metastatic form, thereby decreasing cancer-related deaths especially in 
developing countries where cervical cancer is still the second major cause of death among women.

Finally, it is important to highlight that our investigation, in the Syrian population, is limited to a small number of cases located in a single region of Syria; therefore, it is essential to perform other studies of a larger number of cases from different regions in this country combined with several studies from the Middle East in general. 


\section{Materials and methods}

\subsection{EBV and HPV Detection}

Formalin fixed paraffin embedded blocks of cervical cancer were obtained from 44 Syrian patients with an average age of 57.25 years. Paraffin embedded cervical tumor tissues were obtained from the Department of Pathology, Faculty of Medicine at the University of Aleppo, Syria. The specimens and data used in this study were approved, in May 29, 2008, by the Ethics Committee of the Faculty of Medicine of Aleppo University, \# 2008051, Aleppo, Syria. Five $\mu \mathrm{g}$ of purified genomic DNA (Qiagen GmbH, Hilden, Germany), from each sample, was analyzed for EBV and HPV by PCR using specific primers for LMP1 and EBNA1 as well as E6/E7 of HPV types 16, 18, 31, 33, 35, 45, 51, 52 and 58, while, primers for GAPDH gene were used as an internal control (Tables 1 and 2). This analysis was performed as previously described by our group ${ }^{9,36}$.

\subsection{Tissue microarray}

The tissue microarray (TMA) construction was achieved as illustrated previously by our group 23, 37. Briefly, cervical cancer samples were embedded into a virgin paraffin TMA block using a manual tissue arrayer (Beecher Instruments, Silver Spring, MD). Each block was assembled without previous knowledge of linked clinical or pathological staging information. Two TMA cores of $1.0 \mathrm{~mm}$ in diameter were sampled from a cohort of 44 block tissue samples of Syrian patients diagnosed with cervical carcinomas. Afterwards, $4 \mu \mathrm{m}$ sections were cut and stained with hematoxylin and eosin (H\&E) on the initial slides to verify the histological diagnosis. Next, slides of the completed blocks were used for immunohistochemistry analysis.

\subsection{Immunohistochemistry}

Immunohistochemical (IHC) procedures examining the expression of LMP1, E6 and Id-1 were carried out using standard practices as follows. To analyze the protein expression patterns of LMP1, E6 and Id-1 in TMA slides, each one was deparaffinised in graded alcohol, rehydrated, and boiled (microwave) in $10 \mathrm{mM}$ citrate buffer ( $\mathrm{pH}$ 6.0) for antigen retrieval. Then, TMA slides were incubated for 35 minutes at $37^{\circ} \mathrm{C}$ with primary 
monoclonal and polyclonal antibodies for LMP1 of EBV and E6 of HPV as well as Id-1 (clone 1-4, clone C1P5, sc-488, from Dako and Calbiochem, Canada; as well as Santa Cruz Biotechnology, USA, respectively) using an automated immunostainer (Ventana Medical System, Tuscon, AZ). The automated Ventana Medical System uses an indirect biotin-avidin system with a universal biotinylated immunoglobulin secondary antibody. Afterwards, slides were counterstained with hematoxylin prior to mounting; staining procedures were completed according to the manufacturer's recommendations. Negative controls were obtained by omitting specific primary antibody for LMP1 and E6 as well as specific blocking peptides from Santa Cruz Biotechnology and antibody for Id-1 protein. Following immunohistochemistry, two independent observers examined all TMA slides. The tumors were considered positive for LMP1, E6 and Id-1 onco-proteins if cancer cells exhibited positivity $\geq 1 \%$. In case of LMP1 protein expression (EBV), we also evaluated the presence of viral infection in tumor-infiltrating lymphocytes and stromal cells.

\subsection{Statistical analysis}

Statistical evaluations were done using IBM SPSS Statistics (version 22; SPSS Inc., Chicago, IL, USA) and R. Data were calculated as nonparametric files. We utilized $x^{2}$ test with Yates correction to assess the significance of the association between cancer aggressiveness, Id-1 expression and the co-presence of EBV and high-risk HPVs. 


\section{Acknowledgements}

We would like to thank Mrs. A. Kassab for her critical reading of the manuscript. This work was supported by Qatar University grants \# GCC-2017-002 QU/KU and QUCG-CMED2018\2019-3. 


\section{References}

1. Ala-Eddin Al Moustafa, A. Y., Lina Ghabreau, Ali H. Mohamed and Amal Achkhar Brain Metastases Progression of Breast Cancer. In Breast Cancer - Carcinogenesis, Cell Growth and Signalling Pathways, Gunduz, M. G. a. E., Ed. InTech: 2011.

2. de Martel, C.; Ferlay, J.; Franceschi, S.; Vignat, J.; Bray, F.; Forman, D.; Plummer, M., Global burden of cancers attributable to infections in 2008: a review and synthetic analysis. Lancet Oncol 2012, 13 (6), 607-15.

3. Al Moustafa, A. E.; Al-Awadhi, R.; Missaoui, N.; Adam, I.; Durusoy, R.; Ghabreau, L.; Akil, N.; Ahmed, H. G.; Yasmeen, A.; Alsbeih, G., Human papillomaviruses-related cancers. Presence and prevention strategies in the Middle east and north African regions. Hum Vaccin Immunother 2014, 10 (7), 1812-21.

4. Ala-Eddin Al Moustafa, F. S. C., Noor Al-Antary, ; Yasmee, A., High-risk human papillomaviruses and Epstein-Barr virus presence and crosstalk in human oral carcinogenesis, Development Oral Cancer: Risk factors \& prevention strategies. In Development of Oral Cancer, Moustafa, A.-E. A., Ed. Springer International Publishing: Cham, 2017.

5. Cyprian FS, A.-F. H., Vranic S, Akhtar S, Al Moustafa AE, Epstein-Barr virus and human papillomaviruses interactions and their roles in the initiation of EMT and cancer progression: A brief review. Frontiers in Oncology 2018, 8.

6. Elgui de Oliveira, D.; Muller-Coan, B. G.; Pagano, J. S., Viral Carcinogenesis Beyond Malignant Transformation: EBV in the Progression of Human Cancers. Trends Microbiol 2016, 24 (8), 649-664.

7. Al Moustafa, A. E.; Ghabreau, L.; Akil, N.; Rastam, S.; Alachkar, A.; Yasmeen, A., HighRisk HPVs and Human Carcinomas in the Syrian Population. Front Oncol 2014, 4, 68.

8. $\quad$ Smith, J. S.; Lindsay, L.; Hoots, B.; Keys, J.; Franceschi, S.; Winer, R.; Clifford, G. M., Human papillomavirus type distribution in invasive cervical cancer and high-grade cervical lesions: a meta-analysis update. Int J Cancer 2007, 121 (3), 621-32.

9. Darnel, A. D.; Wang, D.; Ghabreau, L.; Yasmeen, A.; Sami, S.; Akil, N.; Al Moustafa, A. E., Correlation between the presence of high-risk human papillomaviruses and Id gene expression in Syrian women with cervical cancer. Clin Microbiol Infect 2010, 16 (3), 262-6.

10. Yokota, Y., Id and development. Oncogene 2001, 20 (58), 8290-8.

11. Ling, F.; Kang, B.; Sun, X. H., Id proteins: small molecules, mighty regulators. Curr Top Dev Biol 2014, 110, 189-216.

12. Gupta, G. P.; Perk, J.; Acharyya, S.; de Candia, P.; Mittal, V.; Todorova-Manova, K.; Gerald, W. L.; Brogi, E.; Benezra, R.; Massague, J., ID genes mediate tumor reinitiation during breast cancer lung metastasis. Proc Natl Acad Sci U S A 2007, 104 (49), 19506-11.

13. Castanon, E.; Soltermann, A.; Lopez, I.; Roman, M.; Ecay, M.; Collantes, M.; Redrado, M.; Baraibar, I.; Lopez-Picazo, J. M.; Rolfo, C.; Vidal-Vanaclocha, F.; Raez, L.; Weder, W.; Calvo, A.; Gil-Bazo, I., The inhibitor of differentiation-1 (Id1) enables lung cancer liver colonization through activation of an EMT program in tumor cells and establishment of the premetastatic niche. Cancer Lett 2017, 402, 43-51.

14. Gumireddy, K.; Li, A.; Kossenkov, A. V.; Cai, K. Q.; Liu, Q.; Yan, J.; Xu, H.; Showe, L.; Zhang, L.; Huang, Q., ID1 promotes breast cancer metastasis by S100A9 regulation. Mol Cancer Res 2014, 12 (9), 1334-43.

15. Schindl, M.; Oberhuber, G.; Obermair, A.; Schoppmann, S. F.; Karner, B.; Birner, P., Overexpression of Id-1 protein is a marker for unfavorable prognosis in early-stage cervical cancer. Cancer Res 2001, 61 (15), 5703-6. 
16. Hau, P. M.; Tsang, C. M.; Yip, Y. L.; Huen, M. S.; Tsao, S. W., Id1 interacts and stabilizes the Epstein-Barr virus latent membrane protein 1 (LMP1) in nasopharyngeal epithelial cells. PLoS One 2011, 6 (6), e21176.

17. Li, H. M.; Man, C.; Jin, Y.; Deng, W.; Yip, Y. L.; Feng, H. C.; Cheung, Y. C.; Lo, K. W.; Meltzer, P. S.; Wu, Z. G.; Kwong, Y. L.; Yuen, A. P.; Tsao, S. W., Molecular and cytogenetic changes involved in the immortalization of nasopharyngeal epithelial cells by telomerase. Int $J$ Cancer 2006, 119 (7), 1567-76.

18. Vranic S, C. F., Akhtar S, Al Moustafa AE, The role of Epstein-Barr virus (EBV) in cervical cancer: A brief update. Frontiers in Oncology 2018, 8.

19. de Lima, M. A. P.; Neto, P. J. N.; Lima, L. P. M.; Goncalves Junior, J.; Teixeira Junior, A. G.; Teodoro, I. P. P.; Facundo, H. T.; da Silva, C. G. L.; Lima, M. V. A., Association between Epstein-Barr virus (EBV) and cervical carcinoma: A meta-analysis. Gynecol Oncol 2018, 148 (2), 317-328.

20. Khenchouche, A.; Sadouki, N.; Boudriche, A.; Houali, K.; Graba, A.; Ooka, T.; Bouguermouh, A., Human papillomavirus and Epstein-Barr virus co-infection in cervical carcinoma in Algerian women. Virol J 2013, 10, 340.

21. Al Moustafa, A. E.; Foulkes, W. D.; Benlimame, N.; Wong, A.; Yen, L.; Bergeron, J.; Batist, G.; Alpert, L.; Alaoui-Jamali, M. A., E6/E7 proteins of HPV type 16 and ErbB-2 cooperate to induce neoplastic transformation of primary normal oral epithelial cells. Oncogene 2004, 23 (2), 350-8.

22. Al Moustafa, A. E.; Kassab, A.; Darnel, A.; Yasmeen, A., High-risk HPV/ErbB-2 interaction on E-cadherin/catenin regulation in human carcinogenesis. Curr Pharm Des 2008, 14 (22), 2159-72.

23. Al Moustafa, A. E.; Al-Antary, N.; Aboulkassim, T.; Akil, N.; Batist, G.; Yasmeen, A., Co-prevalence of Epstein-Barr virus and high-risk human papillomaviruses in Syrian women with breast cancer. Hum Vaccin Immunother 2016, 12 (7), 1936-9.

24. Kim, N. R.; Lin, Z.; Kim, K. R.; Cho, H. Y.; Kim, I., Epstein-Barr virus and p16INK4A methylation in squamous cell carcinoma and precancerous lesions of the cervix uteri. $J$ Korean Med Sci 2005, 20 (4), 636-42.

25. Santos, N. B.; Villanova, F. E.; Andrade, P. M.; Ribalta, J.; Focchi, J.; Otsuka, A. Y.; Dale Silva, I., Epstein-Barr virus detection in invasive and pre-invasive lesions of the uterine cervix. Oncol Rep 2009, 21 (2), 403-5.

26. Abudoukadeer, A.; Niyazi, M.; Aikula, A.; Kamilijian, M.; Sulaiman, X.; Mutailipu, A.; Abudula, A., Association of EBV and HPV co-infection with the development of cervical cancer in ethnic Uyghur women. Eur J Gynaecol Oncol 2015, 36 (5), 546-50.

27. Aromseree, S.; Pientong, C.; Swangphon, P.; Chaiwongkot, A.; Patarapadungkit, N.; Kleebkaow, P.; Tungsiriwattana, T.; Kongyingyoes, B.; Vendrig, T.; Middeldorp, J. M.; Ekalaksananan, T., Possible contributing role of Epstein-Barr virus (EBV) as a cofactor in human papillomavirus (HPV)-associated cervical carcinogenesis. J Clin Virol 2015, 73, 70-76.

28. Shoji, Y.; Saegusa, M.; Takano, Y.; Hashimura, M.; Okayasu, I., Detection of the EpsteinBarr virus genome in cervical neoplasia is closely related to the degree of infiltrating lymphoid cells: a polymerase chain reaction and in situ hybridization approach. Pathol Int 1997, 47 (8), 50711.

29. Ikeda, J. I.; Wada, N.; Nojima, S.; Tahara, S.; Tsuruta, Y.; Oya, K.; Morii, E., ID1 upregulation and FoxO3a downregulation by Epstein-Barr virus-encoded LMP1 in Hodgkin's lymphoma. Mol Clin Oncol 2016, 5 (5), 562-566. 
30. Lo, A. K.; Dawson, C. W.; Lo, K. W.; Yu, Y.; Young, L. S., Upregulation of Id1 by Epstein-Barr virus-encoded LMP1 confers resistance to TGFbeta-mediated growth inhibition. Mol Cancer 2010, 9, 155.

31. Prates, J.; Franco-Salla, G. B.; Dinarte Dos Santos, A. R.; da Silva, W. A., Jr.; da Cunha, B. R.; Tajara, E. H.; Oliani, S. M.; Rodrigues-Lisoni, F. C., ANXA1Ac(2)(-)(2)(6) peptide reduces ID1 expression in cervical carcinoma cultures. Gene 2015, 570 (2), 248-54.

32. Yasmeen, A.; Bismar, T. A.; Kandouz, M.; Foulkes, W. D.; Desprez, P. Y.; Al Moustafa, A. E., E6/E7 of HPV type 16 promotes cell invasion and metastasis of human breast cancer cells. Cell Cycle 2007, 6 (16), 2038-42.

33. Rajcani, J.; Banati, F.; Szenthe, K.; Szathmary, S., The potential of currently unavailable herpes virus vaccines. Expert Rev Vaccines 2018, 17 (3), 239-248.

34. Luxembourg, A.; Moeller, E., 9-Valent human papillomavirus vaccine: a review of the clinical development program. Expert Rev Vaccines 2017, 16 (11), 1119-1139.

35. Lee, L. Y.; Garland, S. M., Human papillomavirus vaccination: the population impact. F1000Res 2017, 6, 866.

36. Aboulkassim, T.; Yasmeen, A.; Akil, N.; Batist, G.; Al Moustafa, A. E., Incidence of Epstein-Barr virus in Syrian women with breast cancer: A tissue microarray study. Hum Vaccin Immunother 2015, 11 (4), 951-5.

37. Akil, N.; Yasmeen, A.; Kassab, A.; Ghabreau, L.; Darnel, A. D.; Al Moustafa, A. E., Highrisk human papillomavirus infections in breast cancer in Syrian women and their association with Id-1 expression: a tissue microarray study. Br J Cancer 2008, 99 (3), 404-7. 
Table and figure legends

\section{Table 1}

The specific primer sets for LMP1 and EBNA1 genes of EBV used for PCR amplification.

\section{Table 2}

EBV and high-risk HPVs detection in human cervical carcinomas. The co-incidence of these viruses was found in 15 (34\%) samples out of 44 examined by PCR and IHC using specific primers for LMP1, EBNA1 and E6/E7 genes of EBV and high-risk HPVs types $(16,18,31,33,35,45,51,52$ and 58 ,) as well as monoclonal antibodies for LMP1 and E6, as described in the materials and methods section.

\section{Figure 1}

A-B images reflect the diffused and strong cervical cancer cell positivity for HPV (E6 oncoprotein) (A) and EBV (LMP1 protein) (B) (10x); Images C and D highlight HPV and EBV positivity at higher magnification (D); as shown, EBV positivity is clear in some stromal cells and tumor infiltrating lymphocytes (arrows) (D) (20x).

\section{Figure 2}

A case of poorly differentiated (high-grade, non-keratinizing) cervical carcinoma: Upper images highlight the presence of EBV (LMP1 protein), HPV (E6 onco-protein) and Id-1 protein expression (10x); Lower images are respective high-power images (20x); note the presence of EBV-positive tumor infiltrating lymphocytes (arrows). 


\section{Table 1}

\begin{tabular}{lc} 
Genes & \multicolumn{1}{c}{ Primers } \\
\hline LMP1 & 5'-TTGGAGATTCTCTGGCGACT-3' \\
& 5'-AGTCATCGTGGTGGTGTTCA-3' \\
EBNA1-297 & 5'-AAGGAGGGTGGTTTGGAAAG-3' \\
& 5'-AGACAATGGACTCCCTTAGC-3' \\
EBNA1-207 & 5'-ATCGTGGTCAAGGAGGTTCC-3' \\
& 5'-ACTCAATGGTGTAAGACGAC-3' \\
GAPDH & 5'-GAAGGC-CATGCCAGTGAGCT-3' \\
& 5'-CCGGGAACTGTGGCGTGAT-3' \\
\hline
\end{tabular}

\section{Table 2}

\section{Tested cases Detection method $\mathrm{EBV}^{+}$\& $\mathrm{HPV}^{+} \quad$ Percentage}

\begin{tabular}{llllc}
\hline $\begin{array}{c}\text { Cervical cancer tissues } \\
\text { (Paraffin samples) }\end{array}$ & 44 & PCR/IHC* & 15 & 34 \\
\hline
\end{tabular}

\footnotetext{
* These two methodologies, PCR and IHC, were used to detect the presences of EBV and high-
} risk HPVs. 


\section{Figure 1.}
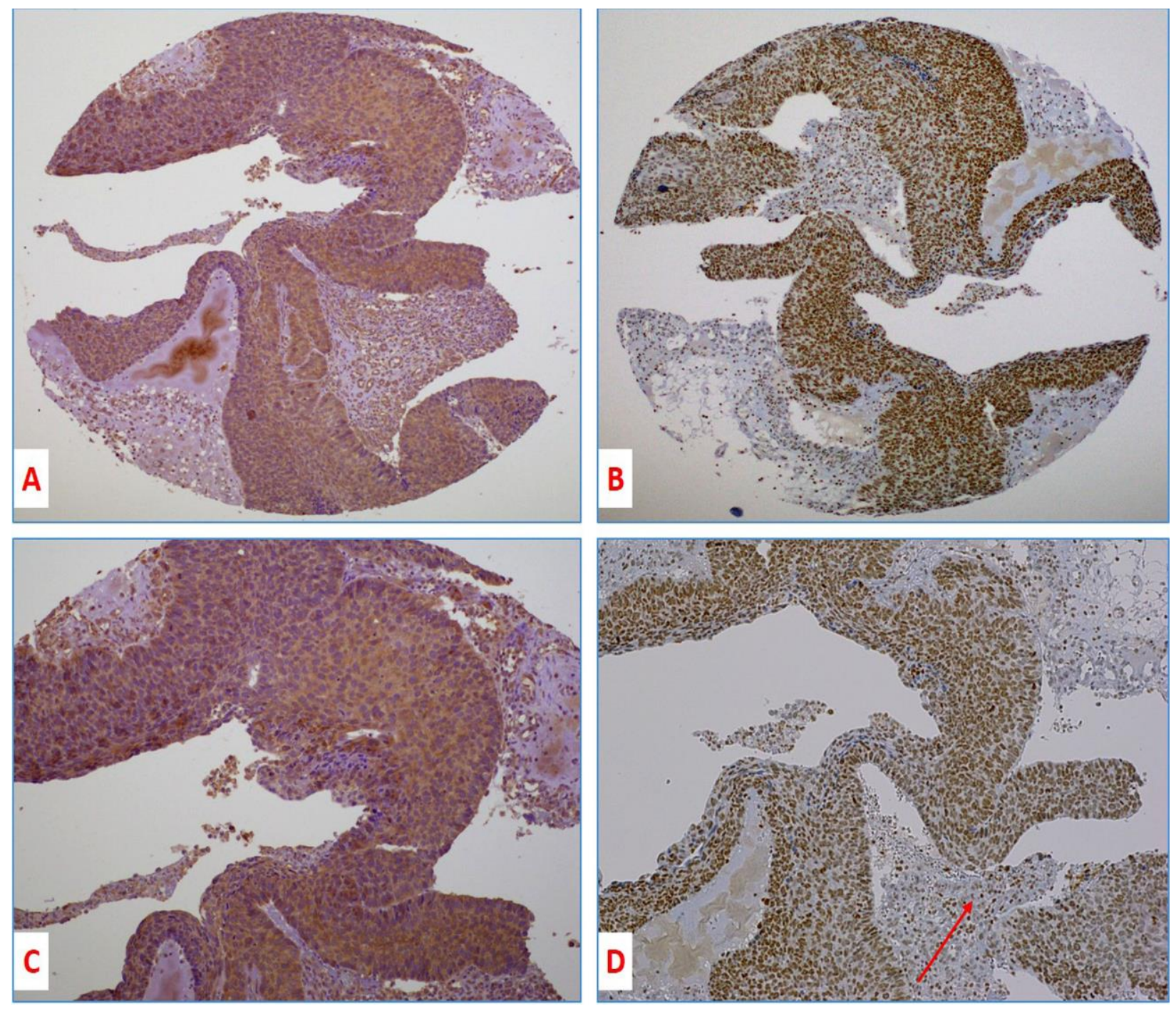


\section{Figure 2.}
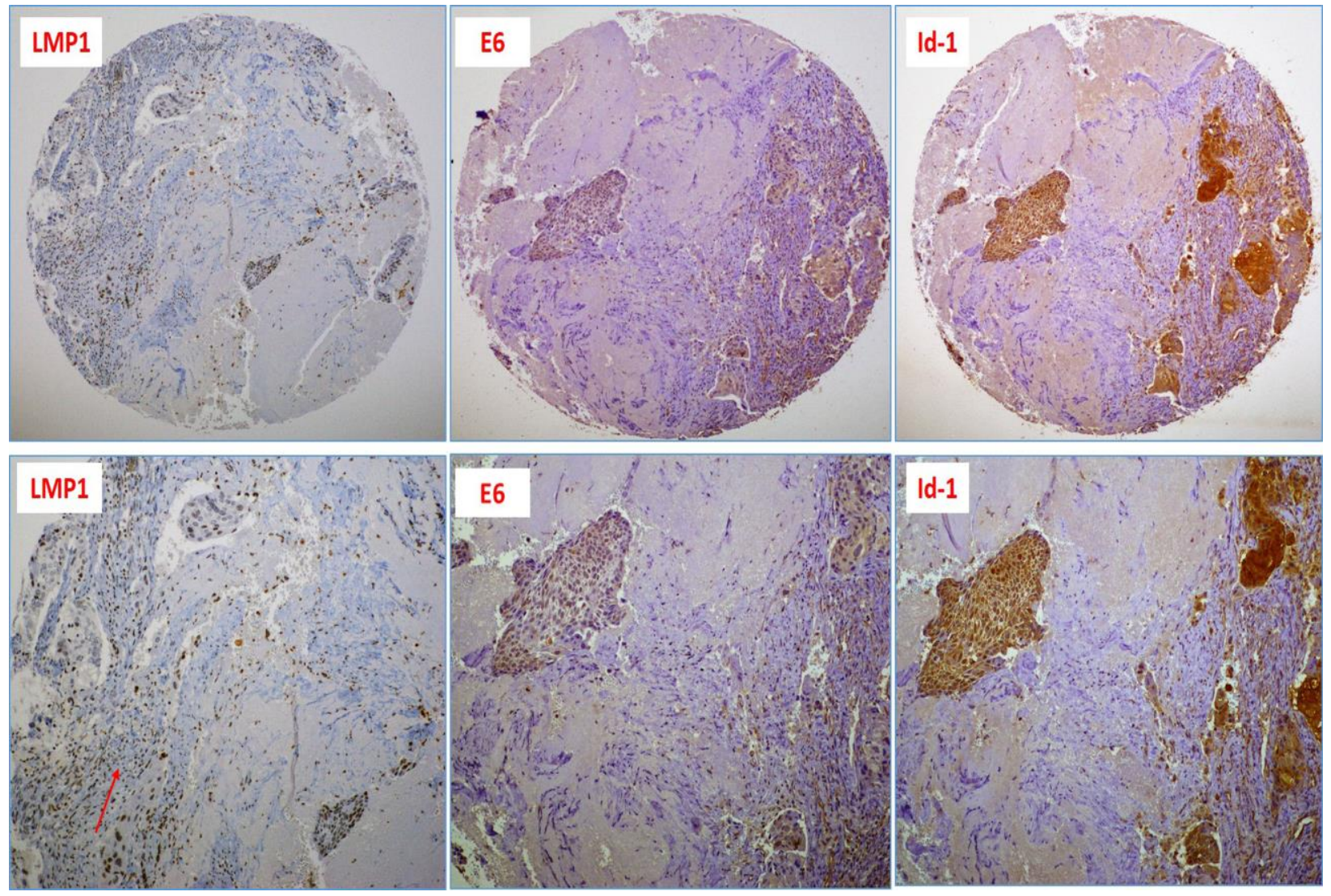